\title{
Transmission Electron Microscopy and Atom Probe Tomography Analysis of Rare-Earth Hexaboride Nanowires
}

\author{
D. R. Diercks, ${ }^{*}$ C.L. Cheung, ${ }^{* *}$ J. Brewer** \\ * Center for Advanced Research and Technology, University of North Texas, Denton, TX 76203 \\ ** Department of Chemistry, University of Nebraska-Lincoln, Lincoln, NE 68588
}

Rare-earth hexaboride $\left(\mathrm{REB}_{6}\right)$ materials possess a number of interesting physical properties including superconductivity [1], semiconductivity [2], fluctuating valence states [3], metal-insulator transitions [4], and low work functions [5] which make them candidates for a variety of applications. Additionally, there is little variation in the crystallographic lattice parameters of the majority of $\mathrm{REB}_{6}$ materials [6], suggesting that these can be grown coherently and free of stress-induced defects. This, then, offers great potential for the tuning of local electronic properties through control of the rare-earth (RE) dopant distribution.

A method for the vapor-liquid-solid growth of $\mathrm{REB}_{6}$ nanowires across a dozen different RE species using a Pd catalyst has been demonstrated through this research. The knowledge of the crystallinity, impurity incorporation, stoichiometry, and uniformity of these nanowires can improve the development and potential implementation of these materials into nanoscale electronic devices. Atom probe tomography (APT) in conjunction with transmission electron microscopy (TEM) allows the atomic scale analysis necessary to discern these important aspects and can be used to provide feedback on the synthesis process. Techniques for such analyses have been previously demonstrated. APT on silicon and III-V nanowires has been used to show the presence of intentional dopants [7] and unintentional dopants [8]. But these analyses have not previously been applied to $\mathrm{REB}_{6}$ nanowires.

In this work, different compositions of $\mathrm{REB}_{6}$ nanowires (including $\mathrm{HoB}_{6}, \mathrm{LaB}_{6}, \mathrm{SmB}_{6}, \mathrm{YB}_{6}$, and $\mathrm{Nd}_{\mathrm{x}} \mathrm{Y}_{1-\mathrm{x}} \mathrm{B}_{6}$ ) were studied using a previously described method [8] for consecutive TEM and APT analysis of nanowires. This allowed for TEM imaging of the nanowires before and after the APT analysis. The mass spectra indicate that only the RE elements and B were present in the bulk of the nanowires. Analysis of the catalyst particles revealed that they were composed of mainly $\mathrm{Pd}$ and $\mathrm{Si}$ in the form of a hexagonal $\mathrm{PdSi}_{\mathrm{x}}$ after the reaction. There were also regions of segregation of the $\mathrm{Si}$ as visible on the right side of both the atom probe reconstructed image and TEM image in Figure 1. Only trace amounts of the La and B were retained in the catalyst. The 1-D concentration profile in Figure 2 shows an approximately 2 nm layer of increased La concentration at the interface between the catalyst and the nanowire. The high resolution TEM image of that same area shows a corresponding disruption in the crystal structure. This increased concentration is likely occurring during the cooling of the system below the liquid eutectic temperature. The incorporation of multiple RE elements within the same nanowire was also explored. Figure 3 shows the mass spectrum from a $\mathrm{Nd}_{\mathrm{x}} \mathrm{Y}_{1-\mathrm{x}} \mathrm{B}_{6}$ nanowire.

References

[1] Hiebl, K.; Sienko, M. J., Inorg. Chem., 19 (1980) 2179.

[2] Mercurio, J. P. et al., Less-Common Met., 47 (1976) 175.

[3] Kebede, A. et al., Phys. B. Condens. Matter, 223 (1996) 256.

[4] Derr, J. et al., Phys. Rev. B, 77 (2008) 193107.

[5] Gesley, M.; Swanson, L. W., Surf. Sci., 146 (1984) 583. 
[6] Schmidt, P. H.; Joy, D. C., J. Vac. Sci. Technol., 15 (1978) 1809.

[7] Perea, D. E. et al., Nat. Nanotechnol., 4 (2009) 315.

[8] Perea, D. E. et al., Nano Lett., 6 (2006) 181.

[9] Diercks, D. R. et al., Microsc. Microanal., 15(Suppl. 2) (2009) 254.
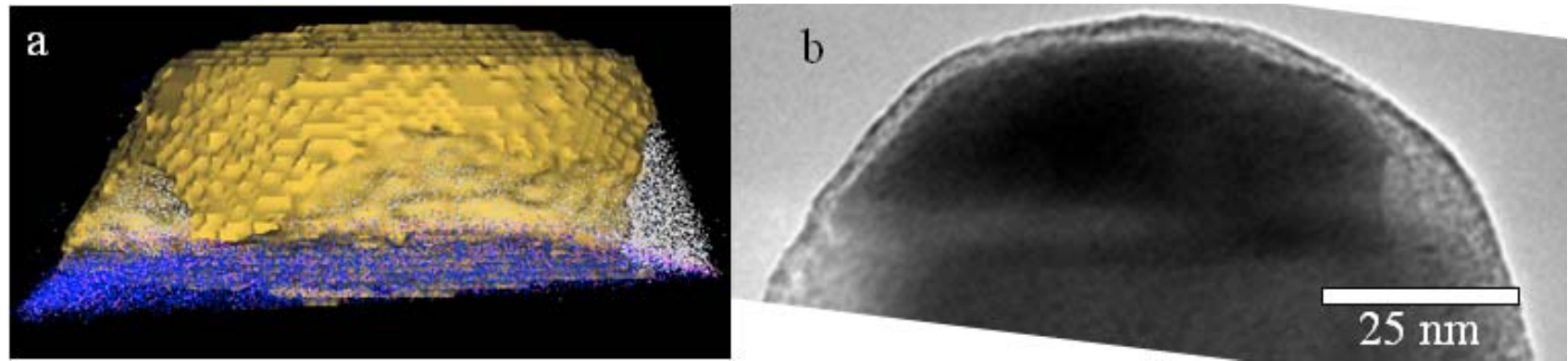

Figure 1 - (a) APT reconstruction of the catalyst particle on a $\mathrm{LaB}_{6}$ nanowire. The silver dots are $\mathrm{Si}$; blue - B; pink - La. Pd is shown as an isoconcentration surface in yellow. (b) TEM image of the same nanowire prior to APT analysis.
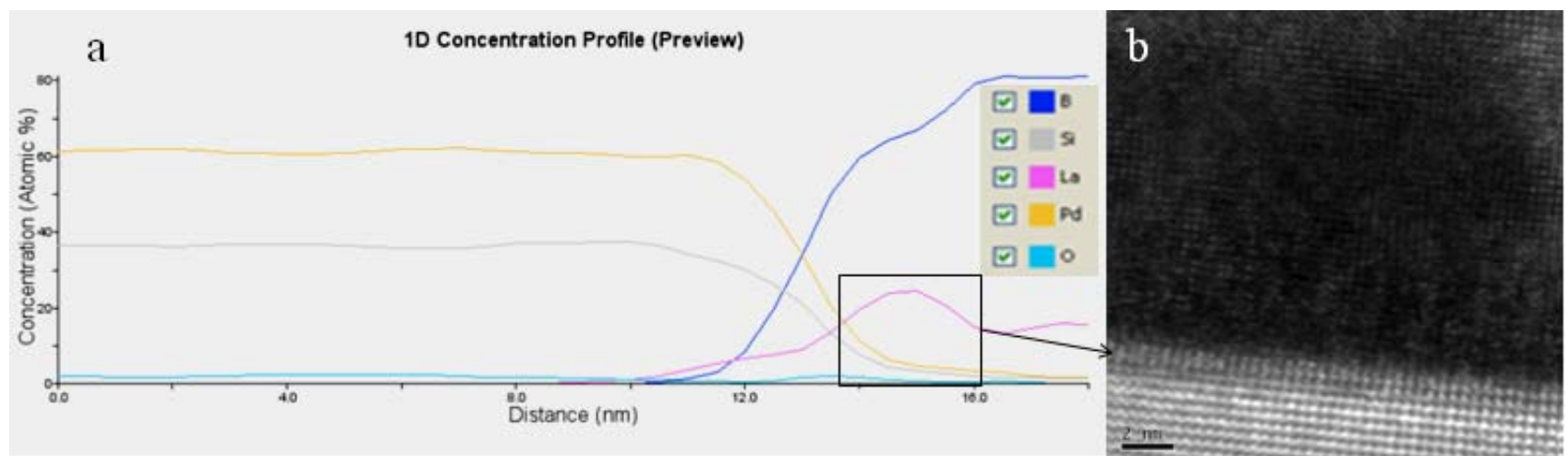

Figure 2 - (a) 1-D concentration profile from the catalyst particle into the nanowire. (b) TEM image of the catalyst/nanowire interface. The box indicates a layer of enhanced La concentration, with an arrow indicating that same layer in the TEM image.

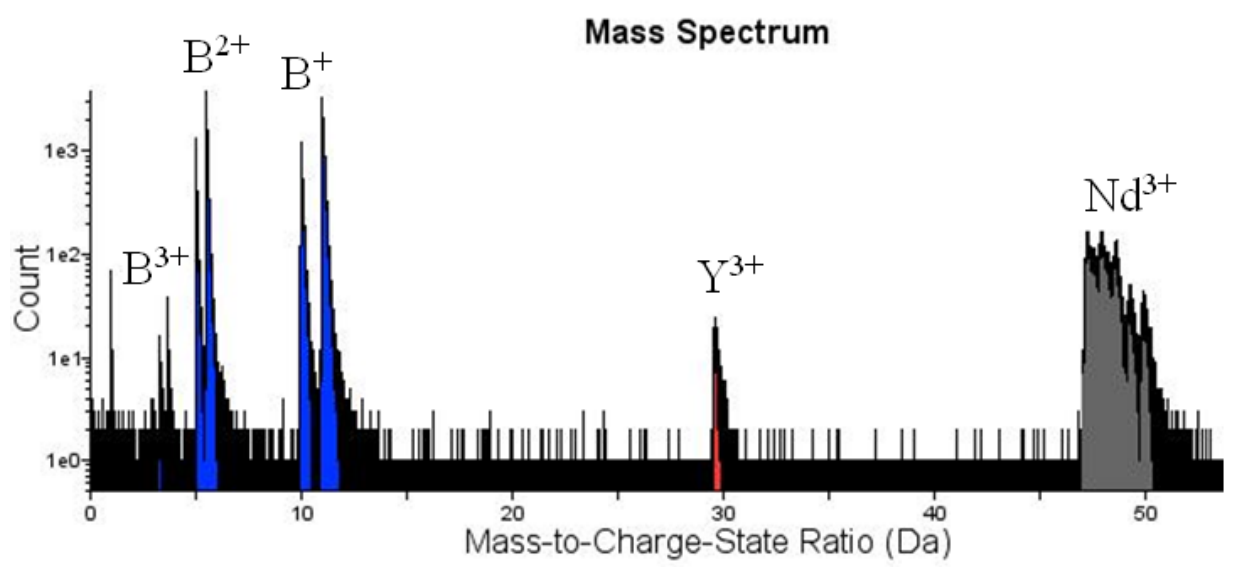

Figure 3 - Mass spectrum from atom probe analysis of a $\mathrm{Nd}_{\mathrm{x}} \mathrm{Y}_{1-\mathrm{x}} \mathrm{B}_{6}$ nanowire. 